\title{
A Redução do Ventrículo Esquerdo não apenas Diminui a Pós-Carga
}

\author{
Paulo J. F. Tucci, Rosemeire M. Kanashiro
}

São Paulo, SP

\begin{abstract}
A proposta, aparentemente insólita, de retirada de fatia do miocárdio para tratamento de cardiopatias com disfunção contrátil avançada, lastreia-se em princípio fisiológico irrefutável: a redução da cavidade ventricular resultante facilita a ejeção ventricular.

Como ocorre com todos os músculos, o miocárdio tem as funções precípuas de desenvolver força e encurtar. Estas duas funções guardam sempre uma relação inversa, isto é, quando o miocárdio é exigido a desenvolver maior força, o encurtamento é prejudicado. Inversamente, quando menos solicitado no desenvolvimento de força, o montante de encurtamento miocárdico se acentua. Este princípio rege a acentuação do deságüe ventricular que acompanha as diminuições da pós-carga.

No coração, em função da distribuição espacial do miocárdio acabar compondo uma cavidade, circunstancialmente, a força desenvolvida pelo músculo é convertida em pressão, que é a variável que regula fluxo e, portanto, regula a ejeção ventricular. As relações entre a força desenvolvida pelo músculo e a pressão gerada no interior da cavidade são definidas pela lei de Laplace: a força miocárdica é diretamente proporcional à pressão intraventricular e ao raio da cavidade e, inversamente proporcional à espessura da pare$\mathrm{de}(\mathrm{F}=\mathrm{P} \times \mathrm{R} / 2 \mathrm{~h})$. Deriva que, para desenvolver uma determinada pressão de ejeção, a força a ser gerada pelo miocárdio é diretamente proporcional à relação raio da cavidade/ espessura da parede, freqüentemente considerada como a relação volume/massa. Reduzindo-se o volume da cavidade, será menor a força a ser gerada pelo miocárdio para desenvolver a mesma pressão de ejeção, configurando a diminuição da pós-carga.

A aplicação destes conceitos às miocardiopatias - e conseqüente indicação de cirurgia para redução da cavida-
\end{abstract}

Laboratório de Fisiologia Cardíaca - Departamento de Fisiologia - Escola Paulista de Medicina - UNIFESP.

Endereço para correspondência: Paulo J. F. Tucci - Rua Estado de Israel 181/94

Cep 04022-000 - São Paulo, SP - E-mail: tucci@fcr.epm.br

Recebido para publicação em 11/8/03

Aceito em 2/9/03 de - gerou expressivo interesse de grupos cirúrgicos internacionais, visando estabelecer o mérito da proposta. Seguindo-se período inicial de otimismo, é perceptível a existência atual de descrença nos resultados da redução do ventrículo esquerdo como estratégia de tratamento das miocardiopatias ${ }^{1}$.

O insucesso da aplicação, contudo, não invalida a tese, e o princípio persiste verdadeiro: cavidades reduzidas facilitam o desenvolvimento de pressão e, conseqüentemente, o esvaziamento ventricular. É cabível lamentar que o rápido abrandamento do interesse pela proposta tenha restringido os conhecimentos fisiopatológicos acrescidos pela prática da redução do ventrículo esquerdo. Equivocadamente, prevalece a idéia de que a diminuição da pós-carga é a única repercussão funcional considerável que resulta da redução da cavidade ventricular².

Avaliando as repercussões funcionais da retirada de cicatriz fibrosa do infarto do miocárdio para a ação mecânica do coração isolado de ratos, foi possível caracterizar, que são bem mais amplas as implicações fisiológicas da diminuição da câmara ventricular ${ }^{3}$. É presumível que esses dados, colhidos experimentalmente, possam auxiliar na compreensão futura das razões que se superpõem aos benefícios funcionais propiciados pela redução da cavidade e terminam por comprometer os resultados nos pacientes submetidos a ventriculectomia parcial. Mais ainda, é desejável que possam contribuir para se discernir o porquê do insucesso quando o princípio é aplicado às miocardiopatas, contrariamente ao verificado com o reconhecido sucesso das reduções da cavidade conduzidas em aneurismectomias.

Os painéis da figura 1 resumem benefícios da redução da cavidade para a função sistólica. Neles, os valores da pressão desenvolvida durante a contração, antes e depois da retirada da cicatriz fibrosa, são projetados em função de três de seus condicionantes: a força parietal gerada durante a contração, a pressão diastólica ventricular e o volume da cavidade. Em A, os valores das pressões desenvolvidas antes e depois da retirada da cicatriz fibrosa do infarto são projetados em função das respectivas forças que as despertaram. Fica patente que, para mesmos valores de força, as pressões desenvolvidas são sistematicamente mais eleva- 


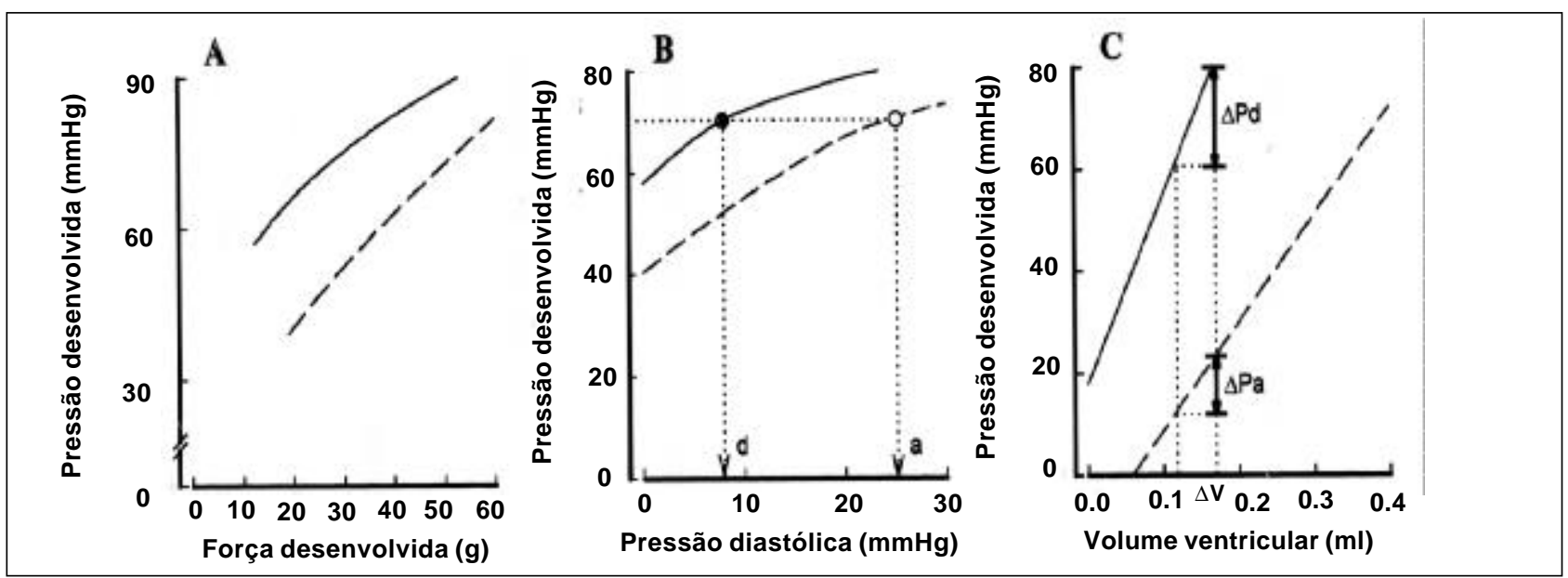

Fig. 1- Influência da redução da cavidade ventricular esquerda na ação mecânica de corações isolados de rato antes (linhas interrompidas) e depois (linhas contínuas) de retirada de cicatriz fibrosa de infarto do miocárdio; A) as pressões desenvolvidas são projetadas em função das respectivas forças geradas durante as contrações, notando-se que, em todos os valores, as pressões desenvolvidas são mais elevadas depois que a cavidade está reduzida; B) as pressões diastólicas compõem o eixo das abcissas. Pressões desenvolvidas mais elevadas ocorrem para mesmos valores de pressões diastólicas. Notar que, depois (d) da retirada da cicatriz fibrosa, um determinado valor de pressão desenvolvida (linha pontilhada) é gerado em níveis de pressões diastólicas inferiores àqueles exigidos antes (a) da redução da cavidade ventricular (setas pontilhadas); C) a capacidade em desenvolver pressão é projetada em função dos volumes ventriculares. As retas refletem como a redução da cavidade ventricular interfere na capacidade miocárdica em gerar pressão, permitindo caracterizar as solicitações do mecanismo de Frank-Starling promovidas pela mesma variação de volume $(\Delta \mathrm{V})$ antes $(\Delta \mathrm{Pa})$ e depois $(\Delta \mathrm{Pd})$ da retirada da cicatriz fibrosa do infarto do miocardio. Evidencia-se que, quando o volume ventricular varia, a menor dimensão da cavidade torna-a apta a acentuar mais a capacidade em gerar pressão.

das depois da redução do ventrículo esquerdo. Estes dados, além de sinalizarem para a redução da pós-carga, colocam em destaque a maior eficiência da câmara reduzida em converter a variável que regula função muscular (força) na variável que regula ejeção ventricular (pressão). Quando a pressão diastólica é utilizada como referencial (painel B), fica patente a maior capacidade em gerar pressão para mesmos valores de pressão diastólica na câmara de menor volume. Mais ainda, as linhas e as setas pontilhadas definem as modificações promovidas pela redução da câmara ventricular nas interações entre as pressões diastólicas e as desenvolvidas. A figura evidencia que, na cavidade de menor volume, mesmos valores de pressão sistólica são alcançados a partir de pressões diastólicas inferiores. Esta influência da redução da cavidade ventricular é especialmente interessante para as circunstâncias. Ao lado das influências benéficas para a função sistólica, a redução da cavidade ventricular restringe a capacidade continente do ventrículo esquerdo, tendendo a promover níveis mais elevados de pressão de enchimento e acentuação da congestão venosa. É perceptível que as relações entre as pressões diastólicas e sistólicas ilustradas em $B$ atuam no sentido de se contrapor ao aumento das pressões de repouso. É provável que seja este o mecanismo que permite entender o intrigante resultado de ejeção ventricular sustentada, ou até mesmo intensificada, com níveis de pressão diastólica ventricular inferiores aos pré-operatórios, após cirurgias de redução do ventrículo esquerdo, segundo vários trabalhos ${ }^{49}$. Finalmente, em C, as pressões desenvolvidas são apresentadas como variável dependente do volume ventricular. Nesta projeção, a curvilinearidade das relações sisto-diastólicas dissipa-se e é possível definir uma reta representativa de cada uma das situações analisadas, permitindo prever o comportamento do mecanismo de Frank-Starling, antes e depois da redução do ventrículo esquerdo. Percebe-se que a reta correspondente aos dados obtidos, quando a câmara tem menor volume, é mais inclinada. É notável que um mesmo aumento do volume ventricular $(\Delta \mathrm{V})$ resultara em intensificação mais expressiva da pressão desenvolvida depois da redução do ventrículo esquerdo $(\Delta \mathrm{Pd}>\Delta \mathrm{Pa})$, indicando que, para uma mesma variacão de volume ventricular, o recrutamento do mecanismo de Frank-Starling é mais intenso na cavidade de menor dimensão.

A expectativa, arrebatada e esperançosa, despertada pela cirurgia de redução do ventrículo esquerdo, nos primeiros tempos que se seguiram à sua divulgação, dificultou $o$ juízo sereno sobre seus méritos. O declínio relativamente rápido de seu prestígio excluiu-a da relação dos temas pesquisados com maior interesse. Condições assim desfavoráveis conspiraram contra o conhecimento adequado da intimidade dos ajustes fisiopatológicos que se seguem à sua concretização. Contudo, os relatos provenientes da aplicação do preceito aos aneurismas ventriculares conduzem a outra conclusão. Os resultados referidos em casos de aneurisma ventricular são suficientemente favoráveis para que seja preconizada a retirada precoce da onerosa cicatriz do infarto do miocárdio ${ }^{10-13}$. Esta outra realidade incita o aprofundamento dos conhecimentos sobre a repercussão funcional da redução da cavidade ventricular, e alerta para a necessidade de se estabelecer as razões pelas quais a cirurgia malogra no tratamento dos miocardiopatas. Os dados atuais apontam como o menor volume ventricular pode favorecer a função cardíaca não só por reduzir a pós-carga. As cavidades menores facilitam o esvaziamento ventricular por acentuarem o recrutamento do mecanismo de Frank-Starling e desviam as relações entre as pressões de enchimento e de ejeção, atenuando ou sobrepujando a tendência para hipertensão diastólica na câmara reduzida. 


\section{Referências}

1. Starling RC, McCarthy PM. Partial left ventriculectomy: sunrise or sunset? Eur J Heart Failure 1999; 1: 313-7.

2. Kass DA. Ventriculectomy. A direct application of Laplace's law. Arq Brasil Cardiol 1996; 67: 401-2.

3. Kanashiro RM, Nozawa E, Murad N et al. Myocardial infarction scar plication in the rat: cardiac mechanics in an animal model for surgical procedures. Ann Thorac Surg 2002; 73: 1507-13.

4. Bocchi EA, Belloti G, Moraes AV et al. Clinical outcome after left ventricular surgical remodeling in patients with idiopatic dilated cardiomyopathy referred for heart transplantation. Short-term results. Circulation 1997; 96: 165-72.

5. Dowling RD, Koening SC, Ewert DL et al. Acute cardiovascular changes of partial left ventriculectomy without mitral valve repair. Ann Thorac Surg 1999; 67: 1470-2.

6. Gradinac S, Miric M, Popovic Z et al. Partial left ventriculectomy for idiopathic dilated cardiomyopathy: early results and six-month follow-up. Ann Thorac Surg 1998; 66: 1963-8.

7. Moreira LF, Stolf N, Bocchi EA et al. Partial left ventriculectomy with mitral preservation in the treatment of patients with dilated cardiomyopathy. J Thorac Cardiovasc Surg 1998; 115: 800-7.
8. Popovic Z, Milutin M, Gradinac S et al. Effects of partial left ventriculectomy on left ventricular performance in patients with nonischemic dilated cardiomyopathy. J Am Coll Cardiol 1998; 32: 1801-8.

9. Scheuder JJ, Steendijk P, van der Veen FHet al. Acute and short-term effects of partial left ventriculectomy in dilated cardiomyopathy. J Am Coll Cardiol 2000; 36 2104-14.

10. Coltharp WH, Hoff SJ, Stoney WS et al. Ventricular aneurysmectomy. A 25-year experience. Ann Surg 1994; 219: 707-14

11. Elefteriades JA, Solomon LW, Mickleborough LL et al. Left ventricular aneurysmectomy in advanced left ventricular dysfunction. Cardiology Clinics 1995 13: 59-72.

12. Sinatra R, Macrina F, Braccio $M$ et al. Left ventricular aneurysmectomy: comparison between two techniques; early and late results. Eur J Cardio-Thorac Surg 1997; 12: 291-7.

13. Taniguchi K, Sakurai M, Takahashi T et al. Postinfarction left-ventricular aneurysm: regional stress, function, and remodeling after aneurysmectomy. J Thorac Cardiovasc Surg 1998; 46: 253-9.

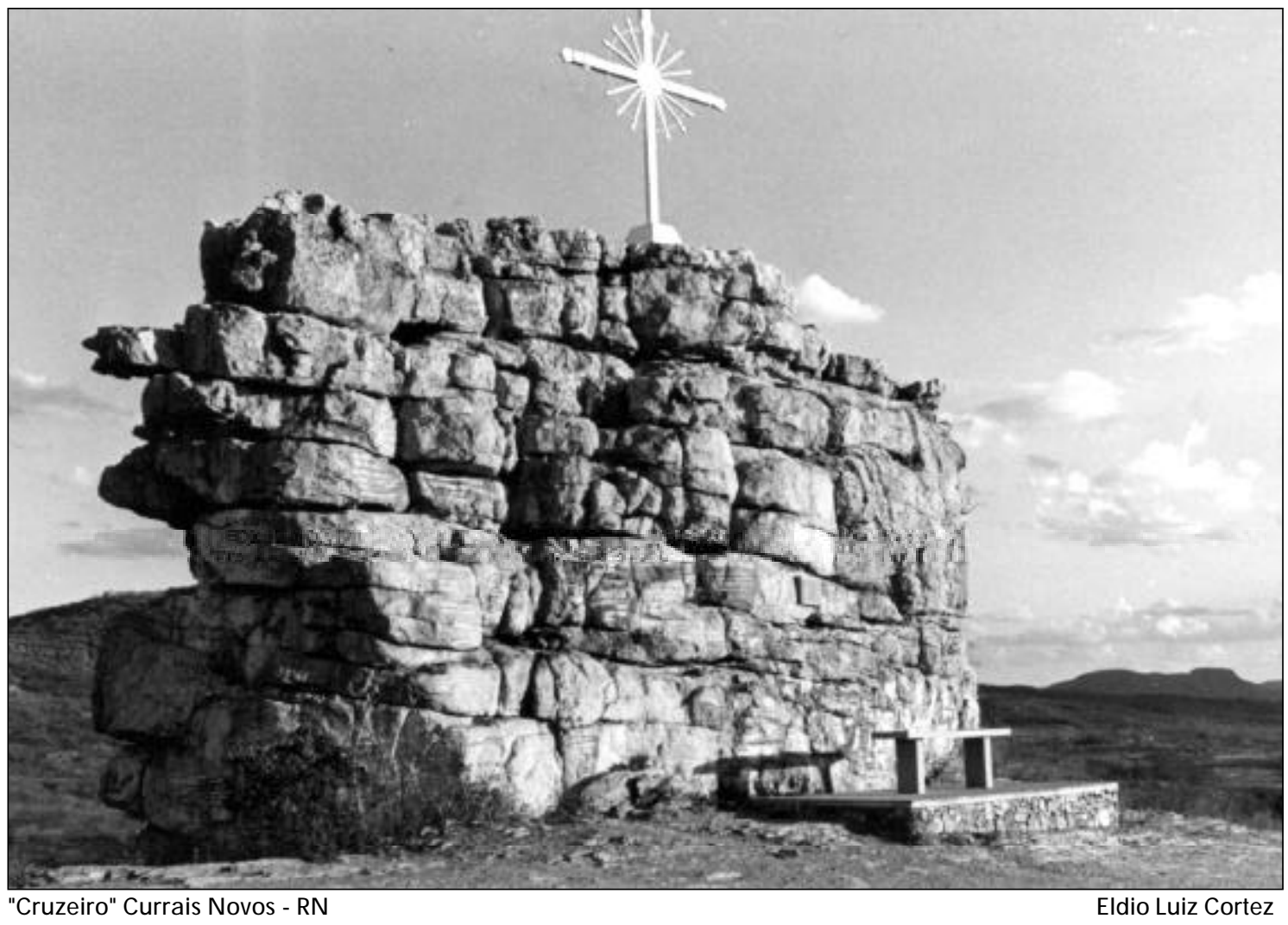

Editor da Seção de Fotografias Artísticas: Cícero Piva de Albuquerque

Correspondência: InCor - Av. Dr. Enéas C. Aguiar, 44 - 05403-000 - São Paulo, SP - E-mail: delcicero@incor. usp.br 